\title{
Efficacy of Pneumatic Retinopexy in Selected Cases of Rhegmatogenous Retinal Detachment
}

\author{
Ejaz Ahmad Javed, ${ }^{1}$ Hamza Subhani, ${ }^{2}$ \\ 1Department of Ophthalmology, Faisalabad Medical University, Allied Hospital, Faisalabad Pakistan, 2MBBS Student, Independent Medical College, Faisalabad-Pakistan
}

\section{ABSTRACT}

Objective: To determine the final anatomical success (Retinal attachment) after pneumatic retinopexy in patients having rhegmatogenous retinal detachment with superior breaks. Study Design: Quasi experimental study. Settings: Ophthalmology Department Allied Hospital (Faisalabad Medical University) Faisalabad Pakistan. Duration: Two years \& 6 moths from January 2016 to June 2018. Methodology: This study was done on 78 diagnosed cases of rhegmatogenous retinal detachment with superior breaks. These cases underwent Pneumatic Retinopexy (PR) by injecting $0.5 \mathrm{ml}$ of SF6 in the vitreous cavity and Cryopexy to the break in the same setting followed by proper positioning. Final anatomical success (Retinal attachment) after pneumatic retinopexy in patients having rhegmatogenous retinal detachment with superior breaks was recorded in 69 patients (88.5\%). Results: Pneumatic Retinopexy showed $88.5 \%$ success in selected cases of rhegmatogenous retinal detachment and $11.5 \%$ failure after this procedure. Conclusion: The post-operated anatomical and functional results were excellent so favoring this technique to be applied as primary procedure for repair of Rhegmatogenous retinal detachment (RRD).

Keywords: Rhegmatogenous retinal detachment, Pneumatic retinopexy efficacy, SF6 Gas.

Corresponding Author

Submitted for Publication: 29-01-2020

Accepted for Publication: 11-04-2020

Dr. Ejaz Ahmad Javed, Associate Professor of Ophthalmology, Faisalabad Medical University, Allied Hospital, Faisalabad-Pakistan.

Email: example@mail.com

Citation: Javed EA, Subhani H. Efficacy of Pneumatic Retinopexy in Selected Cases of Rhegmatogenous Retinal Detachment. APMC 2020;14(2):126-30.

DOI: $10.29054 / A P M C / 2020.861$

\section{INTRODUCTION}

Currently the scleral buckling (SB), Pars plana- vitrectomy (PPV) and pneumatic Retinopexy (PR) are three modalities which are being used to treat Rhegmatogenous Retinal detachment (RRD). ${ }^{1}$

Pneumatic Retinopexy is a safe and cost-effective procedure among all of the above three. ${ }^{2}$

This procedure is associated with less morbidity, reduced cost and faster post-operative recovery as compared to pars plana vitrectomy and scleral buckling. ${ }^{3}$

Treatment of retinal detachment is very essential and should be treated as early as possible as untreated retinal detachment may lead to substantial, severe, and permanent vision loss that might be accompanied by painful hypotony and phthisical eye. ${ }^{4}$ The different clinical trials and series which were used to repair retinal detachment, showed comparable success rate, so these conclusive factors were helpful in choosing this cost suitable technique for certain patients of retinal detachment. ${ }^{5}$

Pneumatic retinopexy is used in $14 \%$ to $17 \%$ cases of RRD making it the 2nd most popular primary modality after PPV. ${ }^{6}$

In the 1980s, pneumatic retinopexy was first introduced and practiced by Dominguez in Spain and later popularized by Hilton and Gizzard. ${ }^{7}$

The Pneumatic Retinopexy (PR) is an office based, nonincisional procedure that has become a well-accepted alternative to scleral buckling and vitrectomy for repair of retinal breaks /break confined to the superior clock hours, within 1-2 clock hours, absence of proliferative retinopathy grade $\mathrm{C}$ or $\mathrm{D}$, a good cooperative patient and clear media. ${ }^{8}$

There are three cardinal steps for repairing a retinal detachment as induction of retinopexy around all retinal breaks with Cryotherapy, then intra ocular gas injection and lastly proper postoperative positioning to provide appropriate gas tamponade to achieve closure of the retinal breaks. ${ }^{9}$

The bubble expansion of Sulfur hexafluoride (SF6) is between 24 to 36 hours after injection and lasts for 6-12 days, however bubble expansion of perfluoro propane (C3F8) bubble is achieved after 3 days of injection and lasts for 38 days. Tensile strength is achieved within 7-14 days following Cryopexy or laser retinopexy. ${ }^{10}$

This procedure is associated with reduced morbidity, reduced cost and faster post-operated recovery as compared to pars plana vitrectomy and scleral buckling. ${ }^{3}$

In a study, success of pneumatic retinopexy was observed in $86 \%$ patients. ${ }^{11}$ While in another study, it was found in $52.3 \%$ of the patients. ${ }^{12}$ By Malik et al the success of pneumatic retinopexy was found in $66 \%$ patients. ${ }^{13}$

The sulfur hexafluoride (SF6) and perfluoro propane (C3F8) are two commonly used intravitreal gases for PR. The Success has been reported with even sterile room air as well. ${ }^{14}$

The intra-ocular bubble behaves on three principle features i.e. buoyancy, surface tension and isolation of retinal tears from intraocular currents. Buoyancy causes upward pressure on the detached retina. The surface tension helps in closing the break and also prevents the bubble to migrate from into sub-retinal 
space. When break is closed then the pump of retinal pigment epithelium removes the sub-retinal fluid. ${ }^{15}$ The final success rate requires the criteria of three factors e.g. presence of a single or multiple retinal breaks occupying one clock hour of retinal arc, superior retinal break at 8 clock hour of globe and ability of the patient to maintain the proper head position for at least 16 hours per day for 5 days or longer. ${ }^{16}$

\section{METHODOLOGY}

Study Design: Quasi experimental study.

Settings: Department of Ophthalmology, Allied Hospital (Faisalabad Medical University), Faisalabad Pakistan.

Duration: Two years \& 6 moths from Jan 2016 to Jun 2018.

Sample Technique: Non probability purposive sampling.

Sample Size: 78 cases of rhegmatogenous retinal detachment. Inclusion Criteria: All patients with shallow superior retinal detachments with breaks at 10 to 2 ' 0 ' clock position of the eye. Minimal PVR or mild tobacco dust, clear cornea, no previous history of retinal reattachment surgery or severe end stage glaucoma.

Exclusion Criteria: All cases with history of severe or end stage glaucoma, tractional retinal detachment or extensive lattice degeneration, retinal detachment with proliferative vitreoretinopathy grade $C$ or $D$, uveitis or vitritis, previous failed pneumatic retinopexy, presence of retinal breaks in the lower 4 clock hours of inferior retinal quadrants and those having to travel to altitude soon after the surgery were excluded from the study.

Methods: Improvement in VA was defined as a gain of two or more lines or final visual acuity better than pre-operative or base line visual acuity. The anatomical outcome was defined as the achievement of a flat retina after a single operation or secondary outcome as achievement of anatomical flat retina at final follow up period.

The eye was anesthetized with $0.3 \mathrm{ml}, 2 \%$ xylocaine subconjunctival injection and supplemented with topical anesthesia by eye drops alcain (proparacaine hydrochloride). Periocular skin was cleaned with povidone iodine and additionally a drop of povidone iodine was instilled in the conjunctival sac. A sterile drape placed over the eye and then a wire speculum was inserted to keep the eye opened. While visualizing with binocular indirect ophthalmoscope, trans-conjunctival cryo was applied. About $0.2 \mathrm{ml}$ aqueous was removed by paracentesis sufficient in lowering of IOP.

A one $\mathrm{ml}$ syringe with detachable needle of 30 gauge was chosen, then needle was detached and syringe was filled with SF6 gas about $0.8 \mathrm{cc}$ with passive pressure, $0.3 \mathrm{cc}$ of the filled gas was discarded with attached needle leaving $0.5 \mathrm{cc}$ of gas. The needle inserted via the pars plana, $4 \mathrm{~mm}$ posterior to limbus in phakic and $3.5 \mathrm{~mm}$ posterior to limbus in pseudophakic patient while facing into center of vitreous cavity. Then patient's head position was adjusted. Then optimum head position of patient was demonstrated to the patient and his or her attendant. The patient was advised to maintain proper position for 7 days. The patient was given a course of oral antibiotics for 5 days with topical antibiotics, steroids and cycloplegic drops for 4 weeks.
Every patient was re-examined at post-operative period of 1 day, 1 week, 2 weeks 8 weeks and 12 weeks intervals.

Laser photocoagulation was done on 2-3 day when needed.

SPSS version 20 was used for data analysis of this study. The mean and SD were calculated for all quantitative variables like age. The frequency and percentage were calculated for all qualitative variables like gender, lens status, complications, vision and success. Effective modifiers like age, lens status, and gender were controlled by stratification. Post-stratification chisquare test was applied. $\mathrm{P}$ value $\leq 0.05$ was considered statistically significant.

\section{RESULTS}

Age distribution of the patients was done, it shows that $57.7 \%$ $(n=45)$ were between 18 to 40 years of age whereas $42.3 \%$ $(n=33)$ were between 41 to 65 years of age, mean \pm SD was calculated as $39.72 \pm 14.123$ years. (Table 1 )

Table 1: Age and gender distribution with lens status

\begin{tabular}{|l|l|l|l|}
\hline \multicolumn{2}{|l|}{ Baseline Characteristics (n=78) } & Frequency & Results \\
\hline \multirow{3}{*}{ Age } & $18-40$ years & 45 & $(57.7 \%)$ \\
\cline { 2 - 4 } & $41-65$ years & 33 & $(42.3 \%)$ \\
\hline \multirow{2}{*}{ Gender } & Male & 55 & $(70.5 \%)$ \\
\cline { 2 - 4 } & Female & 23 & $(29.5 \%)$ \\
\hline \multirow{3}{*}{ Lens Status } & Phakic & 47 & $(60.3 \%)$ \\
\cline { 2 - 4 } & Pseudophakic & 31 & $(39.7 \%)$ \\
\hline
\end{tabular}

For age $=\mathrm{SD}(39.72+-14.123) \quad$ Chi-square value $=2.473$

For Gender $=$ Chi-Square value $=6.764$

Gender distribution shows that $70.5 \%(n=55)$ were male and $29.5 \%(n=33)$ were females (Table 2$)$

Out of 69 effective cases, 52 (94.5\%) were male and 17(73.9\%) were females; $p$ value was 0.009 showing no significant difference. (Table 2)

Table 2: Distribution of success

\begin{tabular}{|l|l|l|l|l|}
\hline \multicolumn{2}{|l|}{ Variable } & Success & Failure & P Value \\
\hline \multirow{2}{*}{ Age } & $18-40$ years & $42(93.3 \%)$ & $3(6.7 \%)$ & \multirow{2}{*}{0.116} \\
\cline { 2 - 5 } & $41-65$ years & $27(18.8 \%)$ & $6(18.2 \%)$ & \\
\hline \multirow{2}{*}{ Gander } & Male & $52(94.5 \%)$ & $3(5.5 \%)$ & \multirow{2}{*}{0.009} \\
\cline { 2 - 5 } & Female & $17(51.5 \%)$ & $6(26.1 \%)$ & \\
\hline \multirow{2}{*}{$\begin{array}{l}\text { Lens } \\
\text { Status }\end{array}$} & Phakic & $47(100 \%)$ & - & \multirow{2}{*}{0.0001} \\
\cline { 2 - 5 } & Pseudophakic & $22(70.97 \%)$ & $9(29.3 \%)$ & \\
\hline
\end{tabular}




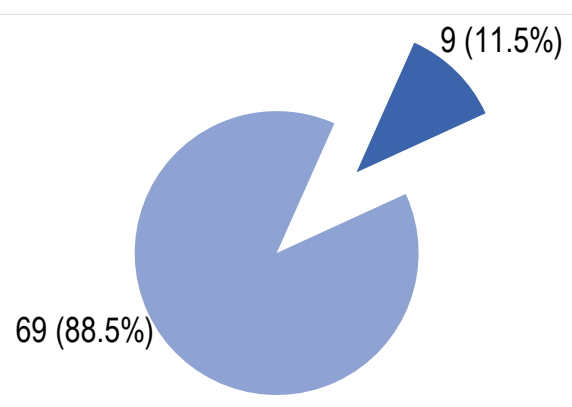

Failure

Success

Figure 1: Frequency of success of pneumatic retinopexy in patients having rhegmatogenous retinal detachment with superior breaks

The follow-up period was as a schedule of postoperative $1^{\text {st }}$ day, 1 st week, $2^{\text {nd }}$ week, 8 th week and $12^{\text {th }}$ week. The 56 eyes (71.8\%) out of 78 had single break in 9 to 2 clock hours of eye while 22 eyes $(28.2 \%)$ had $2-3$ breaks in 9 to 3 clock hours of the globe. (Table 3)

Table 3: Variable findings, complications and procedures

\begin{tabular}{|l|l|l|}
\hline Variables & $\begin{array}{l}\text { No. of patients } \\
\text { (Frequency) }\end{array}$ & Percentage \\
\hline Single break & 56 & $71.8 \%$ \\
\hline 2-3 breaks & 22 & $28.2 \%$ \\
\hline Cryopexy + gas + lasers & 37 & $47.4 \%$ \\
\hline Cryopexy + gas + no lasers & 41 & $52.6 \%$ \\
\hline Hyphema & 1 & $1.28 \%$ \\
\hline Raised IOP & 15 & $19.23 \%$ \\
\hline Limbal wound opening & 1 & $1.28 \%$ \\
\hline Sausage sign & 0 & 0 \\
\hline Suprachoroidal gas & 0 & 0 \\
\hline Subconjunctival gas & 0 & 0 \\
\hline Fish eggs & 10 & $27.12 \%$ \\
\hline Vitreous incarceration & 5 & 6.41 \\
\hline Cataract formation & 8 & $10.26 \%$ \\
\hline Macular hole & 2 & $2.56 \%$ \\
\hline Macular oedema & 4 & $5.13 \%$ \\
\hline Epiretinal membrane & 0 & 0 \\
\hline PVR & 12 & $15.38 \%$ \\
\hline Endophthalmitis & 1 & $1.28 \%$ \\
\hline New or missed breaks & 2 & $2.56 \%$ \\
\hline Re-opening of breaks & 0 & 0 \\
\hline Gas in anterior chamber & 0 & 0 \\
\hline Sub-retinal gas & 0 & 0 \\
\hline
\end{tabular}

\begin{tabular}{|l|l|l|}
\hline $\begin{array}{l}\text { Snellen } \\
\text { vision }\end{array}$ & $\begin{array}{l}\text { No. of patients Pre- } \\
\text { Operative (frequency) }\end{array}$ & $\begin{array}{l}\text { No of Patients Post- } \\
\text { Operative (Frequency) }\end{array}$ \\
\hline $6 / 24$ & - & $1(1.3 \%)$ \\
\hline $6 / 36$ & - & $4(5.1 \%)$ \\
\hline $6 / 60$ & $1(1.3 \%)$ & $33(44.3 \%)$ \\
\hline $4 / 60$ & $20(25.6 \%)$ & $28(35.9 \%)$ \\
\hline $1 / 60$ & $57(73.1 \%)$ & $4(5.1 \%)$ \\
\hline Worst & & $8(10.3 \%)$ \\
\hline Total & 78 & 78 \\
\hline
\end{tabular}

Cryopexy and gas injection was done in all 78 patients, while Cryopexy, gas injection and laser photocoagulation were done in 37 patients $(47.4 \%)$. In 41 patients $(52.6 \%)$ no lasers was used. (Table 4)

\section{DISCUSSION}

The Rhegmatogenous retinal detachment is considered as a commonest retinal emergency which may cause loss of vision. ${ }^{17}$ Pneumatic retinopexy is not used in routine practice for treatment of Rhegmatogenous retinal detachment. As there is controversy in literature regarding the success of pneumatic retinopexy in cases of Rhegmatogenous retinal detachment, so the results of this study may help in creating awareness for the better treatment of Rhegmatogenous retinal detachment by using pneumatic retinopexy with high success.

In this study, out of 78 cases $(n=45) 57.7 \%$ were between 18 to 40 years of age whereas $(n=33) 42.3 \%$ were between 41 to 65 years of age, mean \pm SD was calculated as $39.72 \pm 14.123$ years, whereas $(n=55) 70.5 \%$ were male and $(n=23) 29.5 \%$ were females.

The frequency of success of pneumatic retinopexy in patients having Rhegmatogenous retinal detachment with superior breaks was recorded in $(n=69) 88.5 \%$. Our findings are comparable with the findings of Malik et al who described success of pneumatic retinopexy in $66 \%$ patients. ${ }^{13}$ Whereas another study ${ }^{11}$ recorded $86 \%$ success which also corresponds to our results however, another study ${ }^{12}$ showed only $52.3 \%$ success rate which is lower than the recorded in current study and in other studies. ${ }^{11,13}$

In a study by Assi AC and others ${ }^{18}$ reviewed the management of primary rhegmatogenous retinal detachments during the period 1994 to 1997 by pneumatic retinopexy, the found Pneumatic retinopexy a useful alternative of conventional (retinal detachment surgery).

A Comprehensive Review ${ }^{16}$ during 1986-2007 summarizes surgical outcome for 4,138 eyes for a long period (21 years) similar to those with previous reports where single operational success rate was recorded as $74.4 \%$, final success rate was $96.1 \%$. The new retinal breaks occurred in $11.7 \%$ and proliferative vitro-retinopathy found in $5.2 \%$. They further 
revealed that retinal surgeons must consider all pre-operative ocular features carefully for every individual eye with retinal detachment.

But question is that what is the role of Pneumatic retinopexy in inferior retinal detachments? This study was focusing on superior retinal detachments with superior breaks and I found an answer in an article which was published online by Stephen $S$ and Wing C on march $2018^{3}$ where the researchers described a technique in which the Pneumatic retinopexy was performed and an inverted posture was maintained for 8 hours in nonconsecutive retrospective series of 11 patients and found the single operation success of $82 \%$ in variable centers.

Another wonderful research is found in which bilateral Pneumatic retinopexy was done and a possibility of faster visual rehabilitation was ensured. ${ }^{19}$

This study found some differences in success of pseudophakic or phakic patients rather it may be successful in Pseudophakic/ Phakic patients when break may not be visible. There is a study on 141 eyes undergoing pneumatic Retinopexy with invisible break and no effect on success rate was found. ${ }^{20}$

The pneumatic retinopexy has been used in some very difficult and unusual retinal pathologies. Such study was published in 2012 in European journal of ophthalmology which showed that the Pneumatic retinopexy can be helpful adjunct in cases of retinal detachment which are complicated by hypotony and choroidal detachment. ${ }^{21}$

Pneumatic retinopexy has been used to manage retinal detachments with several other retinal pathologies including Vitelliform macular dystrophy, Cytomegalovirus retinitis, Marfan syndrome and choroidal coloboma. ${ }^{22}$

This retrospective study on 78 classical cases of superior break rhegmatogenous retinal detachment has comparative postoperative complications and results with study by Dhami A et al 2018. ${ }^{23}$

The pneumatic retinopexy occasionally requires brief follow up as compared to other surgical procedures. However, pneumatic retinopexy is easy to perform safer, faster, and cheaper than those with other surgical alternatives. This procedure has short learning curve.

There are Very few intra-operative complications where are recorded in patients treated with pneumatic retinopexy. Improper procedure of gas injection and inaccurate postoperative positioning of patients may cause some complications. The rate of these complications is recorded up to $4 \%$ of the cases $^{18}$ whereas post- operative complications are rarely seen.

It is very astonishing to see the previous reports of Petrushkin et al who stated the successful results of PR in cases of failed scleral buckling and vitrectomies by him. He reported more than $90 \%$ success rate with a secondary Pneumatic retinopexy after failed retinal detachment surgery. ${ }^{24}$

Davis et al reported a success rate of $57 \%$ in Pseudophakic eyes while rate in phakic eyes was $67.5 \%{ }^{25}$ This result is also comparable to results of this study which also showed higher success rate in phakic eyes.
However, Rootman et al found in different studies that Pseudophakic eyes got more Pneumatic retinopexy failures as compared to the other eyes. ${ }^{26}$

\section{CONCLUSION}

In our set up pneumatic retinopexy is not used in routine practice for treatment of rhegmatogenous retinal detachment. However, the results of this study are very encouraging. So, this technique is a safe, easy, faster and cheaper.

\section{LIMITATIONS}

Study was done on rhegmatogenous retinal detachment with only superior breaks and none of cases were included having retinal breaks other than the superior location.

\section{SUGGESTIONS / RECOMMENDATIONS}

The rhegmatogenous retinal detachment having breaks at various clock hours of the eye should be tried as a pneumatic retinopexy procedure.

\section{CONFLICT OF INTEREST / DISCLOSURE}

I have not conflict of interest in this research work.

\section{ACKNOWLEDGEMENTS}

The author acknowledges the patients, nursing staff, operation theatre assistants and research staff of Ophthalmology Department of Allied Hospital / Faisalabad Medical University, Faisalabad.

\section{REFERENCES}

1. Cohen E, Zerach A, Mimouni M, Barak A. Reassessment of pneumatic retinopexy for primary treatment of rhegmatogonous retinal detachment. Clin Ophthalmol. 2015;9:2033-7.

2. Goldman DR, Shah CP, Heier JS. Expanded criteria for pneumatic retinopathy and potential cost savings. Ophthalmol. 2014;121(1):318-26.

3. Stewart S, Chan W. Pneumatic retinopexy: patient selection and specific factors. Clin Ophthalmol. 2018;12:493-502.

4. Chang JS, Smiddy WE. Cost-effectiveness of retinal detachment repair. Ophthalmology. 2014;121(4):946-51.

5. Goldman DR, Shah CP, Heier JS. Expanded criteria for pneumatic retinopexy and potential cost savings. Ophthalmology. 2014;121(1):318-26.

6. Hwang JC. Regional practice pattern retinal detachment repair in the United states. Am J Ophthalmol. 2012;153(6):1125-8.

7. Ellakwa AF. Long term results of pneumatic retinopexy. Clin Ophthalmol. 2012;6:55-9.

8. Fabian ID, Kinori M, Efrati M, Alhalel A, Desatinik H, Hai OV, et al. Pneumatic retinopexy for the repair of primary rhegmatogenous retinal detachment: a 10-year retrospective analysis. JAMA Ophthalmol. 2013;131(2):166-71.

9. Wu L. Pneumatic Retinopexy. [online]. Updated: Jun 04, 2019. Available from: emedicine.medscape.com/article/1844217. overview.

10. Hazzazi MA, Rashaed SA. Outcomes of Pneumatic Retinopexy for the Management of Rhegmatogenous Retinal Detachment at a Tertiary Care Center. Middle East Afr J Ophthalmol. 2017;24(3):143-7. 
11. Mikhail MA, Mangioris G, Casalino G, McGimpsey S, Sharkey J, Best $R$, et al. Outcome of primary rhegmatogenous retinal detachment surgery in a tertiary referral centre in Northern Ireland - a regional study. Ulster Med J. 2017;86(1):15-9.

12. Ling J, Noori J, Safi F, Eller AW. Pneumatic retinopexy for rhegmatogenous retinal detachment in pseudophakia. Semin Ophthalmol. 2018;33(2):198-201.

13. Malik IQ, Iqbal CJ, Afzal Y, Ch N, Mahju TM. Pneumatic retinopexy for early rhegmatogenous retinal detachment. Pak J Ophthalmol. 2015;31(4):203-6.

14. Sebag J. Tang M. Pneumatic retinopexy using only air. Retina. 1993;13(1):8-12.

15. Daniela A. Brinton. Allen Chiang: pneumatic retinopexy. Pneumatic Retinopexy. 2012. Chapter 103.

16. Chan CK, Lin SG, Nuthi AS, Salib DM. Pneumatic retinopexy for the repair of retinal detachment A comprehensive review (19862007). Surv Ophthalmol. 2008;53(5):443-78.

17. Feltgen $\mathrm{N}$, Walter $\mathrm{P}$. Rhegmatogenous retinal detachment--an ophthalmologic emergency. Dtsch Arztebl Int. 2014;111(1-2):1221.

18. Assi AC, Charteris DG, Pearson RV, Gregor ZJ. Pneumatic retinopexy in the treatment of primary rhegmatogenous retinal detachment. Eye (Lond). 1999;13(6):725-8.

19. Kerimoglu H, Ozkagnici A, Okudan S. Bilateral rhegmatogenous retinal detachment repaired with simultaneous bilateral pneumatic retinopexy. Can J Ophthalmol. 2009;44(2):210.

20. Goldman DR, Shah CP, Heier JS, Expanded criteria for PR and potential cost saving. Ophthalmology. 2014;121(1):318-26.

21. Yeung L, Kokame GT, Brord RD, Lightman DA, Lai JC. Pneumatic retinopexy for retinal detachment associated with severe choroidal detachment. Retina. 2011;31(1):87-92.

22. Giansanti F, Giuntoli M, Mazzini C, Pieretti G, Abbruzzese G, Menchini $U$. Pneumatic retinopexy for retinal detachment associated with choroidal coloboma. Eur $\mathrm{J}$ Ophthalmol. 2012;22(4);680-2.

23. Dhami A, Shah KK, Ratra D. Pneumatic retinopexy outcomes as primary or secondary surgical option for treating rhegmatogenous retinal detachment. Indian J Ophthalmol. 2018;66(3):420-5.

24. Petrushkin HJ, Elgohary Ma, Sullivan PM. Rescue pneumatic retinopexy in patients with failed primary retinal detachment surgery. Retina. 2015;35(9):1851-9.

25. Davis MJ, Mudvari SS, Shott S, Rezaei KA. Clinical characteristics affecting the outcome of pneumatic retinopexy. Arch Ophthalmol. 2011;129(2):163-6.

26. Rootman DB, Luu S, M Conti S, Mandell M, Devenyi R, Lam WC, et al. Predictors of treatment failure for pneumatic retinopexy. Can J Ophthalmol. 2013;48(6):549-52.

\footnotetext{
AUTHORSHIP CONTRIBUTION

Ejaz Ahmad Javed

Data Collection, Manuscript Writing, Statistical Analysis and Surgical Skill of the Procedure

Hamza Subhani

References Layout
} 